SELF ASSESSMENT ANSWERS

\section{Postcystoscopy confusion}

\section{Q1: What is the most likely cause for} this patient's acute confusional state?

The patient has an acute episode of confusional state on the first postoperative day with no clinical evidence of focal neurological deficit. Laboratory investigations revealed a sudden drop in serum sodium, which is the most probable aetiology for his acute confusional state.

\section{Q2: What further investigations/ calculations would help in your management?}

For further management of this case, serum osmolarity and osmolar gap should be calculated. They can be approximately calculated from the following formulas:

$$
\begin{aligned}
& \text { Osmolarity }(\mathrm{mOsm} / \mathrm{l})=2\left(\mathrm{Na}^{+}+\mathrm{K}^{+}\right)+ \\
& \text {glucose }+ \text { urea } \\
& \text { Osmolar gap }(\mathrm{mOsm} / \mathrm{l})=\text { measured } \\
& \text { osmolarity }- \text { calculated osmolarity }
\end{aligned}
$$

In our case, the calculated osmolarity was $257.3 \mathrm{mmol} / \mathrm{l}$ and the osmolar gap was $6.7 \mathrm{mOsm} / \mathrm{l}$ (normal $<10 \mathrm{mOsm} / \mathrm{l}$ ). Other investigations like arterial blood gas analysis to evaluate the severity of the metabolic acidosis and serum ammonia levels should be done.

\section{Q3: How would you manage this case?}

Management should be tailored to the individual case depending upon the severity and relative duration of the onset of confusion, serum osmolarity, osmolar gap, serum ammonia levels, and renal function of the patient. In our case, as the hyponatraemia is severe $(<120 \mathrm{mmol} / \mathrm{l})$ and symptomatic with normal osmolar gap and renal function, intravenous 3\% sodium chloride (hypertonic saline) should be given to correct the serum sodium. ${ }^{12}$ Correction of the serum sodium should be gradual and carefully undertaken. The most important adverse effect related to rapid reversal of serum sodium is development of central pontine myelinosis.

\section{Discussion}

A $1.5 \%$ glycine solution, which is slightly hypotonic compared with serum (200 mOsm/ 1), is the most commonly used irrigant during urological procedures like cystoscopy or transurethral resection of prostate (TURP) and gynaecological procedures like endometrial ablation. Hyponatraemia has been well reported as a complication of using hypotonic glycine as the irrigant. One should be aware of the various pathophysiological mechanisms of the development of hyponatraemia in these patients so as to effectively treat them.

Although these solutions are continuously aspirated during cystoscopy, a small amount of fluid can be absorbed through the venules along the bladder wall. In addition, a ruptured prostatic capsule or lacerated urinary bladder can promote increased glycine absorption. Absorbed glycine initially remains in the extracellular compartment but being an osmotically active agent, glycine attracts water from the intracellular space and produces a dilutional hyponatraemia and a raised osmolar gap. ${ }^{3}$ Thus, during the initial phase, when large part of glycine remains in the extracellular compartment, the amount of solution absorbed during the irrigation determines the severity of hyponatraemia. With greater absorption volumes (greater than 3 litres) hypervolumic hyponatraemia or water intoxication occurs. A small to moderate amount of absorbed glycine extracts more intracellular water increasing the osmolar gap and causing dilutional hyponatraemia or glycine toxicity. Later, glycine is eventually transported into the intracellular space and undergoes breakdown into its various metabolites like creatinine, carbon dioxide, water, ammonia, serine, glucose, hippurate, glyoxylate, formate, and oxalate. Renal excretion of glycine, glycine metabolites, and excess extracellular free water subsequently adjusts electrolytes and serum osmolarity back toward baseline values. During this corrective or late phase glycine metabolites, particularly ammonia, may cause ammonia toxicity. ${ }^{4}$

Clinical manifestations during the initial phase of glycine toxicity causing hypervolumic hyponatraemia or water intoxication include headache, visual disturbances, restlessness, initial hypertension followed by hypotension, bradycardia, agitation, confusion, coma, and death. ${ }^{5}$ Osmotic haemolysis can lead to anaemia and thrombocytopenia. Severe metabolic acidosis due to glycine metabolites like hippurate, glyoxalate, and formate can also occur. Hypocalcaemia, which may be severe, can result from the formation of complexes of calcium and oxalic acid. Transient visual disturbances is not a uncommon symptom and may be due to direct neurotoxicity of glycine. Patients with smaller amounts of irrigant absorbed are usually asymptomatic.

An important guide to treat patients is to classify them on the basis of their electrolyte and osmolar status. The most important laboratory abnormality is hyponatraemia with or without a raised osmolar gap. Metabolic encephalopathy may be related to hyponatraemia, hypo-osmolarity, or hyperammonaemia. Asymptomatic patients with serum sodium concentrations $>120 \mathrm{mmol} / \mathrm{l}$ usually respond to simple discontinuation of glycine infusions. If serum sodium concentrations are $<120 \mathrm{mmol} / \mathrm{l}$ or symptoms of glycine toxicity are present, the serum osmolar gap should guide therapy. In patients with a normal serum osmolar gap, hyponatraemia occurs as a result of excess extracellular free water. Correction of hyponatraemia in this setting may require hypertonic saline. Serum sodium should not be corrected faster than 1.5 to $2.0 \mathrm{mmol} /$ hour over 34 hours or $>10 \mathrm{mmol} / \mathrm{l}$ in the first 24 hours and $<18 \mathrm{mmol} / \mathrm{l}$ in the first 48 hours so as to avoid central pontine myelinolysis. In acute symptomatic hyponatraemia, hypertonic saline ( $3 \%$ sodium chloride) is usually given over 3-4 hours and further management is guided by the therapeutic response. ${ }^{12}$ The following formulas will help one to determine the amount of hypertonic saline needed to replenish in acute situations:

$$
\begin{aligned}
& \mathrm{Na}(\mathrm{mmol} \text { given as } 3 \%)=\left(\left[\mathrm{Na}^{+}\right.\right. \\
& \left.\left.(\text {desired })-\mathrm{Na}^{+} \text {(measured) }\right]\right) \times \\
& \text { estimated TBW }
\end{aligned}
$$

TBW (total body water) in a women can be calculated as $0.5 \times$ body weight in kilograms $(\mathrm{kg})$ and in men as $0.6 \times$ body weight in $\mathrm{kg}$.

\section{Learning points}

- Metabolic encephalopathy related to hyponatraemia, hypo-osmolarity, and hyperammonaemia should be suspected in any surgical patient presenting with confusion in the postoperative period after TURP, cystoscopy, or endometrial ablation using glycine as the irrigant solution.

- Glycine toxicity presents with mental status change, hypotension, respiratory depression, haemolytic anaemia, and thrombocytopenia resembling septic shock and disseminated intravascular coagulopathy.

- It is confirmed by the constellation of the following: (a) hyponatraemia, (b) low plasma osmolarity, (c) an increase in osmolar gap, (d) hyperammonaemia, and (e) hypocalcaemia.

- Therapy for glycine toxicity depends on the serum sodium, osmolar gap, and the symptomology. Serum sodium should not be corrected faster than 1.5 to $2.0 \mathrm{mmol} /$ hour over $3-4$ hours or $>10 \mathrm{mmol} / \mathrm{l}$ in the first 24 hours and $<18 \mathrm{mmol} / \mathrm{l}$ in the first 48 hours so as to avoid central pontine myelinolysis.

- Severe glycine toxicity should raise the suspicion for bladder or urethral injury, with the associated risk of infectious complications.

In patients with raised osmolar gaps, hyponatraemia is secondary to glycine itself. Such patients should be considered for haemodialysis to augment renal excretion of glycine and prevent the formation of toxic metabolites. In patients with renal failure, haemodialysis is necessary because they are unable to excrete glycine or free water. ${ }^{6}$ When hyperammonaemia with associated with symtomatology is found, L-arginine infusion, which inhibits the conversion of glycine to ammonia should be considered. ${ }^{7}$ The severity of glycine toxicity is directly related to the amount of glycine absorbed into the systemic circulation. Patients with severe glycine toxicity, therefore, should be evaluated for an underlying bladder rupture or urethral tear that might otherwise be clinically occult.

\section{Final diagnosis}

Hyponatraemia.

\section{References}

Adrogue HJ, Madias NE. Hyponatremia. N Engl J Med 2000;342:1581-9.

2 Kumar S, Berl T. Sodium. Lancet 1998:352:220-8.

3 Gravenstein D. Transurethral resection of the prostate (TURP) syndrome: a review of the pathophysiology and management. Anesth Analg 1997:84:438-46.

4 Shepard RL, Kraus SE, Babayan RK, et al. The role of ammonia toxicity in the post transurethral prostatectomy syndrome. Br J Urol 1987:60:349-51.

5 Olsson J, Nilsson A, Hahn RG. Symptoms of the transurethral resection syndrome using glycine as the irrigant. J Urol 1995;154:123-8.

6 Singer $M$, Patel $M$, Webb $A B$, et al. Management of the transurethral prostate resection syndrome: 
time for reappraisal? Crit Care Med 1990; 18:1479-80.

7 Roesch RP, Stoeling RK, Lingeman JE, et al. Ammonia toxicity resulting from glycine absorption during a transurethral resection of the prostate. Anesthesiology 1983;58:577-9.

\section{A 67 year old woman with renal failure and sinus bradycardia ${ }^{1}$}

\section{Q1: What are the possible causes of renal failure?}

The most likely cause of the renal failure was introduction of the ACE inhibitor for treatment of hypertension. It is possible that this patient could have small vessel atheromatous renovascular disease or even bilateral renal artery stenosis. The renal failure could also be due to diabetes or hypertension, although the speed of progression makes the natural history of either disease unlikely.

\section{Q2: What was the likely cause for the sinus bradycardia?}

In this case, the sinus bradycardia was due to the effect of the increased dose of atenolol, the elimination of which was prolonged by the renal failure, therefore making the drug more potent. Other causes of sinus bradycardia are other drugs (for example, digoxin, verapamil), myocardial infarction, hypothyroidism, hypothermia or, more commonly, age related degeneration of the conduction system (sinoatrial disease). Symptomatic bradycardia associated with syncope or presyncopal episodes requires insertion of a temporary pacing wire and a permanent pacemaker may need to be considered.

\section{Q3: Why did the temporary pacemaker} fail to capture?

The most likely explanation is due to an increase in pacing threshold caused by derangement of serum biochemistry and severe acidosis, rather than inappropriate positioning of the temporary pacing wire. The augmented effect of atenolol may also be a contributory factor to the increase in pacing threshold. The ECG in fig 1 (see $p$ 46) demonstrates failure to capture.

\section{Discussion}

This case illustrates the hazards of drug treatment for hypertension in patients who have mild renal impairment. Worsening of renal failure after initiation of an ACE inhibitor, may indicate underlying bilateral renal artery stenosis, which is usually reversible on stopping the drug. Initial screening is by renal ultrasound or spiral computed tomography scanning and confirmation of diagnosis can be made by either renal arteriography or magnetic resonance angiography. ${ }^{1}$

The elimination half life of atenolol, a hydrophilic $\beta$-receptor blocking drug, which is predominately eliminated via the kidneys, can increase from 6-9 hours to up to 36 hours in patients with renal failure..$^{23}$ The systemic effects of the drug can therefore be enhanced and severe bradycardia may occur. This prolongation of elimination half life requires a dosage adjustment of the drug in patients with renal failure. The effects of $\beta$ blockade are reversed by stopping the drug and giving atropine and isoprenaline. If the blood pressure is low, and cardiac failure is prominent, dobutamine should be started. Intravenous glucagon can be given if hypoglycaemia is a problem. A temporary pacing wire is often required.

Right ventricular pacing is identified by a high frequency pacemaker signal followed by a broad complex QRS configuration demonstrating a left bundle branch block pattern. Due to filtering systems in modern ECG monitoring equipment, it may be necessary to change the chosen lead until an adequate pacing "spike" is seen. Capture can be verified by increasing the heart rate at the pacing box and identifying an appropriate increase in heart rate on the ECG monitor and a similar increase in the pulse rate. The threshold, which is the amplitude in volts needed to maintain capture, can be measured, then doubled to ensure pacing continues in the acute period when the threshold will rise due to trauma at the interface of the tip of the wire and the myocardium. ${ }^{4}$

Failure to capture can occur when the tip of the wire has been placed in an infarcted area or the coronary sinus. It is apparent when a pacemaker signal appears without an appropriate evoked response and the pulse rate does not correspond with the "spikes" seen on the ECG trace (fig l; p 46). Capture thresholds are adversely affected and increased by acidosis, hypoxia, hyperglycaemia, or any other severe metabolic derangement. ${ }^{5}$ Pharmacological intervention with class Ia and Ic antiarrhythmic drugs, verapamil, $\beta$-blockers, hypertonic saline, and glucose-insulin

\section{Learning points}

- ACE inhibitors may precipitate renal failure in patients with underlying small vessel atheromatous renovascular disease or bilateral renal artery stenosis.

- Water soluble $\beta$-blockers - for example, atenolol-are excreted by the kidneys and accumulate in renal impairment; dose reduction is often necessary.

- Treatment by $\beta$-blockers is a common cause of sinus bradycardia, which may reverse on stopping the drug.

- Reversal of $\beta$-blocker effects may require temporary pacing.

- Failure of a temporary pacing wire to capture may be due to biochemical derangement, not operator incompetence!

infusion can also adversely affect capture thresholds. Electrolyte effects tend to be transient, and the pacing threshold may normalise after correction of these disturbances.

\section{Final diagnoses}

(1) Sinus bradycardia secondary to decreased elimination of atenolol therapy in renal failure and (2) temporary pacing wire failure to capture secondary to derangement of serum biochemistry.

\section{References}

1 Williams SG, Lindsay SJ, Tan LB. Recurrent pulmonary oedema in a 53 year old woman. Postgrad Med J 2001;77:408-9.

2 Kirch W, Gorg KG. Clinical pharmacokinetics of atenolol-a review. European Journal of Drug Metabolism and Pharmacokinetics 1982:7:81-91.

3 Wan SH, Koda RT, Maronde RF. Pharmacokinetics, pharmacology of atenolol and effect of renal disease. $\mathrm{Br} J$ Pharmacol 1979:7:569-74.

4 Eellenbogen KA. Cardiac pacing (practical cardiac diagnosis), 2nd Ed. Blackwell Science, 1996.

5 Hazard PB, Benton C, Milnor JP. Transvenous cardiac pacing in cardiopulmonary resuscitation. Crit Care Med 1981;9:666-8. 